\title{
LASER-INDUCED FLUORESCENCE PHOTOGRAMMETRY FOR DYNAMIC CHARACTERIZATION OF TRANSPARENT AND ALUMINIZED MEMBRANE STRUCTURES
}

\author{
Adrian A. Dorrington ${ }^{*}$, Thomas W. Jones ${ }^{\dagger}$, Paul M. Danehy ${ }^{\star}$ \\ Instrumentation Systems Development Branch \\ MS 236, NASA Langley Research Center \\ Hampton, VA, 23681-2199, USA \\ Richard S. Pappa ${ }^{\S}$ \\ Structural Dynamics Branch \\ MS 230, NASA Langley Research Center \\ Hampton, VA, 23681-2199, USA
}

\begin{abstract}
Photogrammetry has proven to be a valuable tool for static and dynamic profiling of membrane based inflatable and ultra-lightweight space structures. However, the traditional photogrammetric targeting techniques used for solid structures, such as attached retro-reflective targets and white-light dot projection, have some disadvantages and are not ideally suited for measuring highly transparent or reflective membrane structures. In this paper, we describe a new laserinduced fluorescence based target generation technique that is more suitable for these types of structures. We also present several examples of non-contact noninvasive photogrammetric measurements of laser-dye doped polymers, including the dynamic measurement and modal analysis of a $1 \mathrm{~m}-\mathrm{by}-1 \mathrm{~m}$ aluminized solar sail style membrane.
\end{abstract}

\section{Introduction}

Gossamer structure research has reached the stage where measurements of the surface profile and dynamic behavior has become an integral part of the design process. Furthermore, measurements of both ground test and in-flight structures is essential for continued gossamer development. Photogrammetry is the science of calculating three-dimensional spatial coordinates from multiple two dimensional photographs. It has proven to be a valuable tool for acquiring these types of measurements. It offers the simplicity of taking photographs coupled with high measurement precision of up to 1 part in 100,000 or higher. ${ }^{1,2}$ Videogrammetry is the extension of still-camera photogrammetric

\footnotetext{
* National Research Council Associate

+ Research Engineer

¥ Research Scientist, Member AIAA

$\$$ Senior Research Engineer, Member AIAA
}

principles to time sequences of images, and is useful for capturing dynamic behavior.

Current practices for photogrammetric measurements of gossamer structures use either attached retro-reflective targets or white light dot projection. Retro-reflective targets provide excellent high-contrast images, but suffer from disadvantages associated with physically attaching targets to the structure. ${ }^{3}$ White-light projection is an alternative that offers the advantage of non-contact measurement, thereby eliminating the problems associated with attached targets. It works well on diffuse material because, by definition, the projected light is scattered in all directions allowing the acquisition of high contrast images from any viewing angle. However, gossamer structures are often manufactured from highly transparent or specularly reflective aluminum-coated materials that provide little diffuse scattering, which in turn causes difficulties when attempting to photograph projected targets. ${ }^{3}$ There are two major disadvantages of white-light projection for these applications. The first is the appearance of hot-spots caused by direct reflections of illumination sources. Secondly, it requires very long exposure times, limiting its use to static measurements.

Laser-induced fluorescence (LIF) target generation has recently been demonstrated as a viable alternative technique for photogrammetric target generation. ${ }^{3.4 .5}$ This method uses LIF to generate diffuse-like scattering that allows image acquisition with fast shutter speeds and high imaging rates, and does not suffer from the disadvantages associated with either attached retro-reflective or white-light projected targets. To date, LIF target generation has been studied and applied to polymer membranes doped with laser-dye to aid the LIF process.

In this paper we first introduce and contrast LIF target generation with traditional retro-reflective and whitelight projection methods. We then present four 
demonstrations of non-contact photogrammetric profiling of dye-doped polymer membranes using the LIF targeting technique. Both static small amplitude wrinkle mapping and dynamic characterization are demonstrated for transparent and aluminized reflective polymers, including a $1 \mathrm{~m}$-by-1m square solar sail style aluminized polymer. These demonstrations highlight the advantages of the LIF based technique, and in some cases, measurements are performed that have previously been unobtainable.

\section{Photogrammetry and traditional photogrammetric targeting techniques}

Photographic images are the result of a perspective projection of a three-dimensional (3D) object onto two dimensions (2D). Consequently, two or more photographic images can be reverse engineered to derive the $3 \mathrm{D}$ shape of the original object. This process is called Photogrammetry, and the solution provides a quantitative relationship between a $3 \mathrm{D}$ object and the $2 \mathrm{D}$ images acquired by the cameras. ${ }^{6}$

While photogrammetry has its roots in the topographic mapping and surveying field, the last two decades have seen close-range photogrammetric techniques developed to support various industrial and research applications. For example, in some areas of aeronautics aeroelastic experimentation, including model deformation and wing twist, photogrammetric measurements have become part of the standard data set. ${ }^{6}$ Originally, the techniques were developed for use with still-frame film cameras, but the rapid development of CCD technology has led to the successful migration from film to digital camera and video technology

Accurate photogrammetric measurements require the photography of high contrast surface features that appear in at least two images, as shown in Figure 1. However, many objects commonly measured do not naturally exhibit such features. Traditionally, retro-

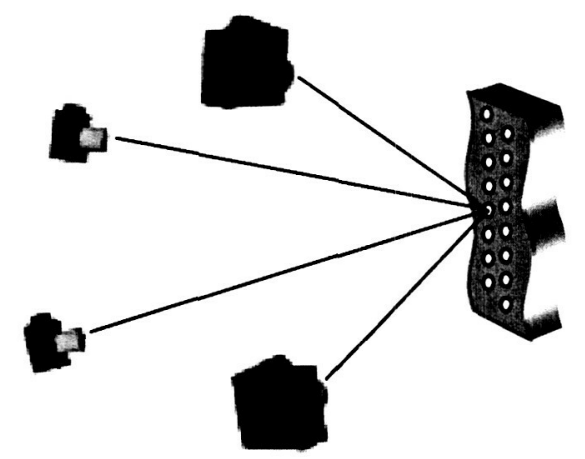

Figure 1 - Multi-camera photogrammetric measurement principle reflective targets are painstakingly attached to the object to artificially provide these high contrast features. When illuminated, with a camera flash for example, these targets reflect light directly back to the illuminating source, causing the targets to appear very bright in contrast to the background.

Retro-reflective targets work exceptionally well and have very few drawbacks when used on solid structures. However, even though they have been used on gossamer structures providing excellent results, $7,8,9$ they introduce several undesirable effects when used on gossamer membrane structures. The primary concerns are added mass and altered stiffness, which in turn alter and mask the true characteristics of the structures. Furthermore, once affixed, the pattern of attached targets cannot be changed and the targets are very difficult or impossible to remove due to the delicate nature of the membrane material. ${ }^{3}$ An alternative solution is to project the target pattern on the structure, thereby providing a completely non-invasive photogrammetric measurement. ${ }^{3}$

Projected dot patterns provide good results with diffuse surfaces because light is scattered in all directions, and the projected pattern can be viewed and photographed over a wide viewing angle. ${ }^{10}$ However, when applied to gossamer structures difficulties arise because the membrane material is usually either highly transparent or reflective (aluminum coated). In both cases, there is very little diffusely scattered light because the majority of light passes directly through a transparent membrane or is specularly reflected off an aluminized membrane. Consequently, exposure times as long as 30 seconds are required to obtain sufficient contrast images from white-light illuminated aluminized membranes. ${ }^{3}$ These long exposure times prohibit the use of white-light projection for dynamic studies, and limit its application to static measurements. Furthermore, no measurements of white-light illuminated transparent membranes have been yet reported.

A further complication arises with photogrammetric observations of reflective structures, using either the retro-reflective or projected targets method, is the appearance of glints or hot-spots. These hot-spots are areas of bright illumination on the image that is generated as a direct reflection of the camera flash or other unrelated source such as room lights. Hot-spots act to reduce the contrast of targets, or in many cases completely occlude the targets, in a localized region of the image rendering these targets unusable. ${ }^{9}$ The problem of hot-spots is illustrated in Figure 2, which shows a dye-doped polymer attached to a reflective surface, simulating an aluminized polymer, illuminated by both a camera flash (with affixed retro-reflective 

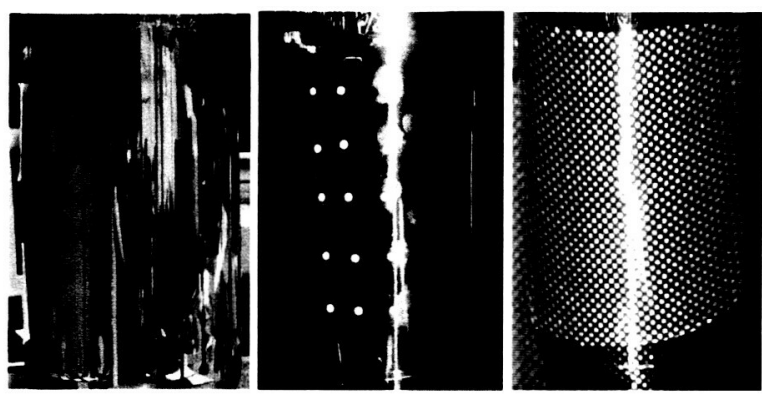

Figure 2 - Transparent dye-doped polymer fixed to a reflective cylinder simulating an aluminized membrane (left), with attached retro-reflective targets and illuminated with a camera flash (center), and illuminated with white-light dot projection (right). Note the hotspots apparent in both illuminated images. Previously reported by Jones et al. ${ }^{4}$

targets) and by white-light dot projection. ${ }^{4}$ Due to the cylindrical nature of the object, the hot-spots appear as bright vertical lines that occlude entire columns of targets.

\section{Laser-induced fluorescence target generation}

Laser-induced fluorescence (LIF) is a process of inelastic ${ }^{*}$ scattering in which a portion of incident laser light is absorbed by a material (solid, liquid, or gas), and is then emitted at a longer wavelength. Many materials exhibit the phenomenon, but some are explicitly designed to utilize and optimize it. One example of such an optimized material is laser dye, which is usually dissolved in methanol and used as the optical gain medium in dye lasers.

In order to utilize LIF as a mechanism to generate diffuse-like scattering in transparent polymer membranes, small quantities of Rhodamine 590 laser dye [Exciton, Dayton, Ohio] have been added to the polymers during their manufacture. When these dyedoped polymers are subjected to laser radiation at or

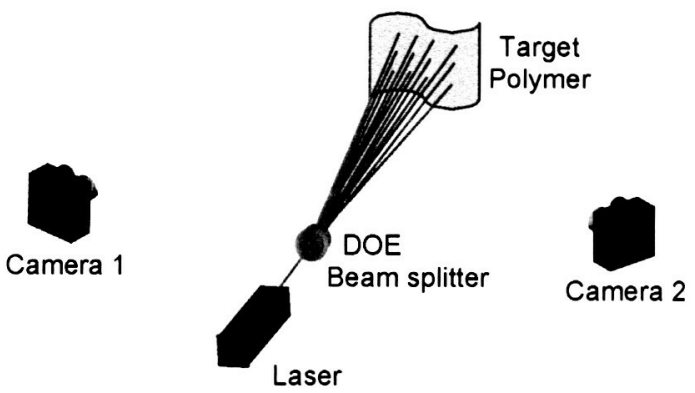

Figure 3 - Laser-induced fluorescence photogrammetry principle.

\footnotetext{
"In optical physics, inelastic scattering is the scattering process in which there is a change of photon energy and hence wavelength. This is opposed to elastic scattering where there is no change in energy or wavelength
}
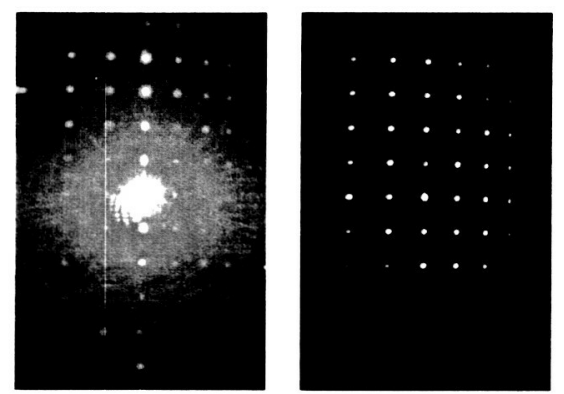

Figure 4 - Transparent dye-doped polymer fixed to a reflective cylinder simulating an aluminized membrane illuminated with a laser dot pattern. Photographed without a filter showing a glint (left), and the same illumination with filter blocking laser light, eliminating the glint, and showing only laser-induced fluorescence (right).

near $532 \mathrm{~nm}$, they fluoresce and emit light in all directions allowing high contrast images to be obtained from almost any viewing angle.

Our previous research investigating the photo-physics of laser dye doped membranes has proven their suitability for photogrammetric target generation. ${ }^{5}$ In particular, the fluorescence emission pattern was found to afford a wider viewing angle than diffuse scatter from white-light projected targets. The laser-dye doped into the polymer was also found to have a substantial useful lifetime, specifically in excess of 200 minutes of continual operation at a frame rate of $25 \mathrm{~Hz}$. In addition, the fluorescence temporal decay time was found to be in the order of nanoseconds. This is several orders of magnitude faster than high-speed photography exposure times, and hence well within the limits required for dynamic videogrammetry.

To generate a fluorescing photogrammetry target pattern, an array of laser beams is projected onto the structure being studied, as shown in Figure 3. The resulting fluorescing target pattern is then photographed by two or more cameras and processed with standard photogrammetry software. A diffractive optical element (DOE) [Stocker Yale, Montreal, Canada] is employed to split a single laser beam source into the required beam array.

An optical filter is used in front of the camera to remove any stray laser light and prevent any hot spots that might otherwise be observed. Figure 4 clearly illustrates the effectiveness of this filter. The same reflective polymer shown in Figure 2 is illuminated with a 7-by-7 laser spot pattern and photographed with and without the optical filter. In the unfiltered image a reflection of one laser beam enters the camera directly causing a massive hot-spot that renders the image completely useless. With the filter installed, the hotspot is eliminated and all targets are visible. For an indepth discussion regarding filter selection and photo- 
physics of the dye-doped polymers, see Dorrington et al. $^{5}$

\section{Measurement Demonstration}

To illustrate the effectiveness of laser-induced fluorescence based photogrammetric profiling, several measurement examples will now be presented. These demonstrations include both a high spatial resolution static measurement and a larger scale dynamic measurement for the two cases of transparent and aluminized films.

\section{Static wrinkles in a transparent polymer}

Transparent polymers have so far proven impossible to measure with white-light projection techniques, which fail to generate sufficient diffuse scatter from the membrane to provide suitably high contrast images. LIF target generation can, however, provide noncontact target generation in dye-doped transparent polymers. Our group has recently reported an experiment designed to demonstrate the ability of LIF based photogrammetry to measure small amplitude wrinkles in a transparent membrane. ${ }^{4}$ This experiment will now be summarized here for completeness.

The experimental set-up is shown in Figure 5. It is very simple and closely follows the principle shown in Figure 3. The article being measured is a 12-inch diameter circular transparent dye-doped polymer membrane, manufactured by SRS Technologies [Huntsville, AL], stretched over a hoop to provide near uniform tension. This hoop has been compressed in one direction to induce small wrinkling.

A low power, $2 \mathrm{~mW}$ green $(543.5 \mathrm{~nm})$ Helium Neon $(\mathrm{He}-\mathrm{Ne})$ laser was used as the illumination source. Its beam passes though a diffractive optical element

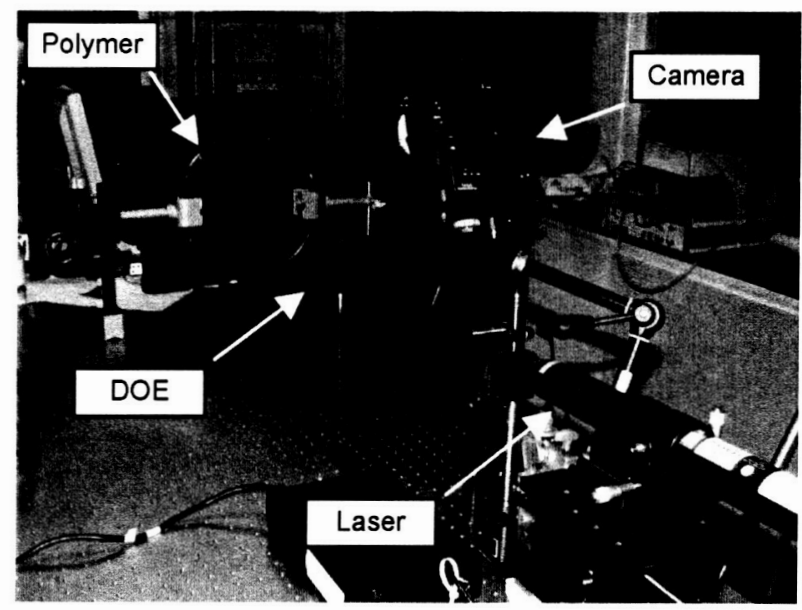

Figure 5 - Experimental set-up for transparent polymer static measurements. ${ }^{4}$ producing a 19x19 grid over the surface of the membrane. Figure 6 shows a typical image of this dot pattern on the membrane. The image is slightly over exposed to clearly show the polymer and support ring. Images used for photogrammetric processing are optimized for highest contrast dot pattern. The size, density, and arrangement of the target pattern can be easily adjusted to fit any test article, with additional optics. Furthermore, the lower intensity second and higher order diffractive dots visible around the edges of the 19-by-19 pattern can be eliminated with spatial filtering if required.

In this example, the target has a high intensity center spot (180 times brighter than any other spot) that is a result of using an inexpensive off-the-shelf DOE that was designed for operating at a different wavelength $(670 \mathrm{~nm})$. Some of the experiments described below use similar DOEs, but later experiments use a custom manufactured DOE designed for the correct wavelength. Our custom manufactured DOEs include patterns with either a center spot of the same intensity as the rest of the pattern, or 3 times brighter for identification purposes.

Photographs were taken from four different viewing angles with a professional grade six mega-pixel Kodak DCS 760M camera fitted with a Kodak \#22 Wratten Gelatin filter to block the laser light. This camera was calibrated to determine all camera parameters and lens distortions prior to performing the measurements. The images were processed in Photomodeler ${ }^{\mathbb{B}}$ [EOS Systems, Vancouver, BC, Canada] software to calculate $3 \mathrm{D}$ coordinates for each target in the pattern, which were plotted in Figure 7 using the Surfer ${ }^{\mathbb{B}}$ software package [Golden Software Inc, Golden, CO].

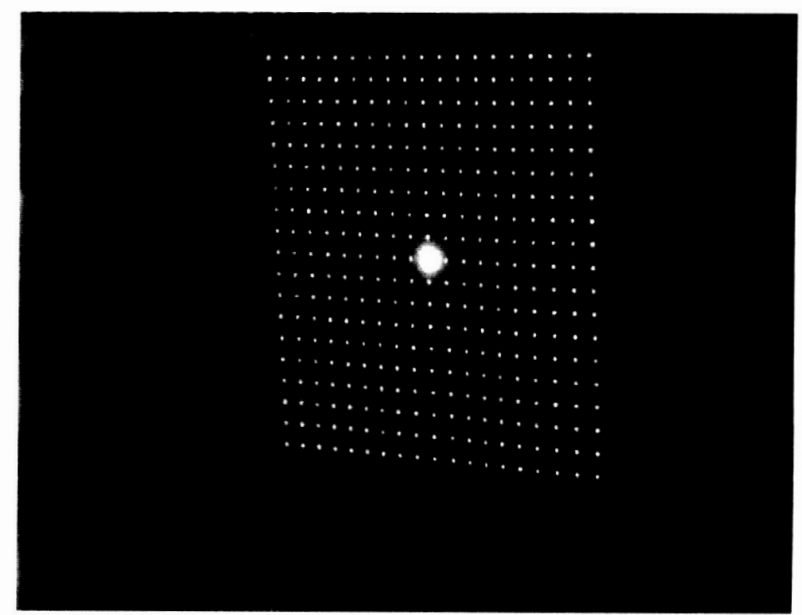

Figure 6 - Fluorescent target pattern on tensioned transparent dyedoped membrane. ${ }^{4}$ 


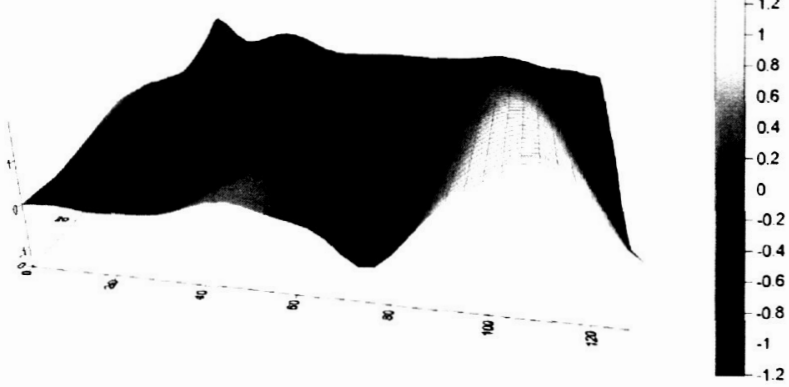

Figure 7 - Shaded relief map overlaid with wire-frame model of wrinkles in compressed membrane. Note the clearly visible nature of the wrinkles despite the low amplitude in the order of $1.5 \mathrm{~mm}$.

This surface plot clearly shows capability of the system to distinguish small amplitude wrinkles in the membrane, in the range $\pm 1.5 \mathrm{~mm}$, with a good signal to noise ratio. Non-contact photogrammetric measurements of wrinkles in aluminized membranes has been demonstrated before at a similar level of detail, ${ }^{9}$ but no such measurements of transparent membranes have been reported.

\section{Dynamics of a transparent polymer}

The previous example demonstrated the capability to perform static measurements of a transparent polymer, which were previously unobtainable using white-light projection techniques. This capability is now developed one step further and a demonstration of a dynamic measurement is presented.

For dynamic measurements the previous experimental set-up is changed in several ways. As shown in Figure

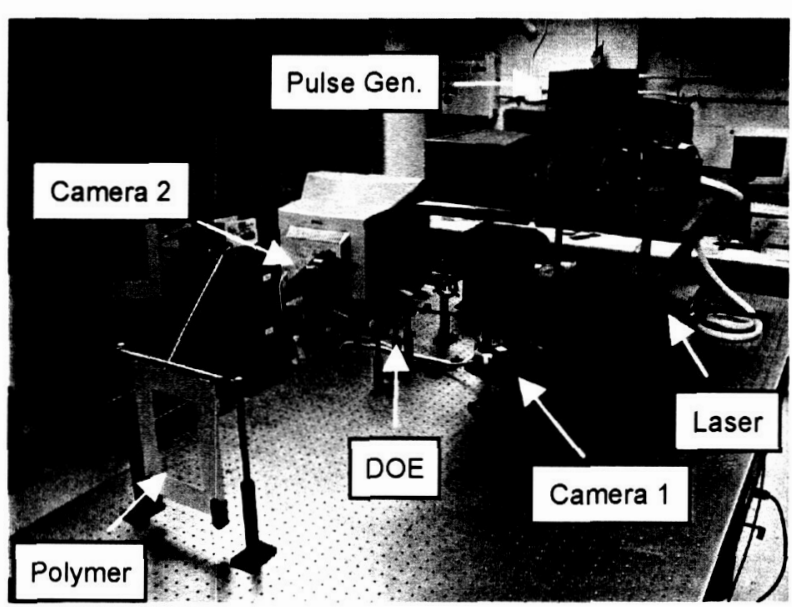

Figure 8 - Experimental set-up for transparent polymer dynamic measurements

8 , the illuminating laser was replaced with a $\mathrm{Nd}$ :YAG pulsed laser source producing $10 \mathrm{~mJ}$ pulses at variable repetition rate up to $30 \mathrm{~Hz}$. For dynamic studies, the pulsed laser is much more appropriate than the $\mathrm{CW}$ laser because of its very short pulse time and consequently high peak power output. The pulsed laser also provides more average power than the Continuous Wave $(\mathrm{CW})$ laser, but this is not its primary advantage. During the static measurements, exposure times of several seconds could be used to combat low fluorescence intensity levels. For dynamic studies, however, short exposure times and short laser pulses are needed to avoid motion blur.

The Nd:YAG laser used in this experiment achieves these goals with a $10 \mathrm{~ns}$ pulse time, which also allows the camera shutter time to be set at a level that rejects a)
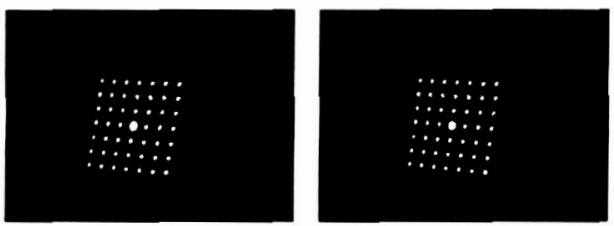

b)
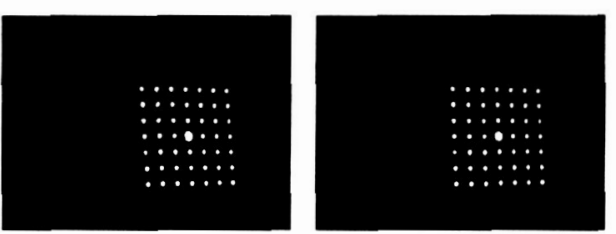
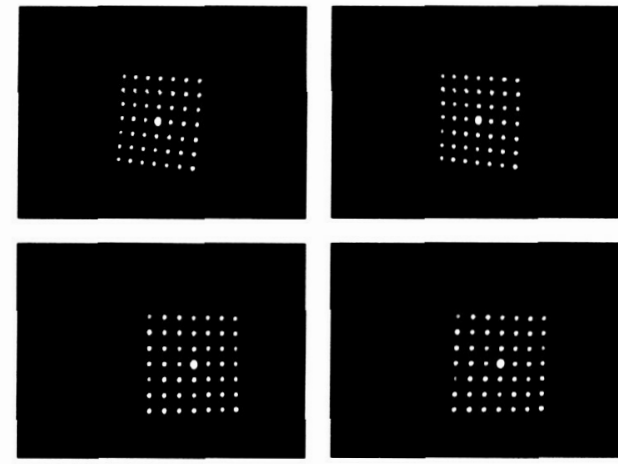
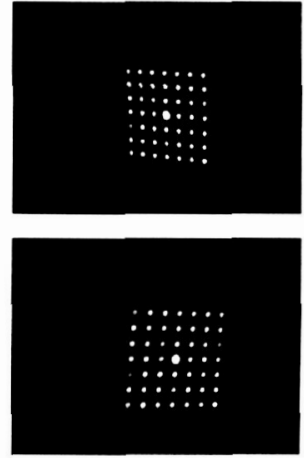

c)

Figure 9 - Time sequence of dynamic measurement of transparent laser-dye doped polymer covering half of a swing cycle. a) Image sequence from camera 1. b) Image sequence from camera 2. c) Animation of computed 3D model (viewed from the side) 
background room light illumination. For comparison, one pulse from the $\mathrm{Nd}: \mathrm{YAG}$ laser contains an equivalent number of photons as a 5 second exposure using the He-Ne laser described previously.

The still camera used in the previous experiment was replaced with two Pulnix TM-7610 video cameras attached to a PC computer fitted with digital video acquisition cards. The laser pulse rate and video camera acquisition rate were controlled and synchronized with a Stanford Research Systems [Sunnyvale, California] pulse generator model DG535.

The test structure was formed by mounting a dye-doped transparent polymer on a card frame suspended from a bar. Weights were attached to the bottom of the frame and the polymer was allowed to swing back and forth freely. Just prior to initiating image acquisition, the weights were lifted and released to induce a swinging motion. Two hundred images were then acquired at a rate of $25 \mathrm{fps}$, covering approximately 7 full swing cycles, and processed in the Photomodeler Video software. A time series showing one half of a swinging oscillation as viewed from both cameras, and a computer model reconstruction are shown in Figure 9.

Figure 10 shows the computed displacement along the axis of pattern projection for two targets over time. The exponential swing amplitude decay can be seen on this plot, along with the differences in amplitude between two targets; one near to the pivot axis, and one at maximum distance from the pivot axis.

From a modal analysis perspective, a more interesting motion is observed when the polymer and frame are excited by an impulse and the bottom-center between the weights, and more than one vibration mode can be observed. Such an excitation was employed in a second

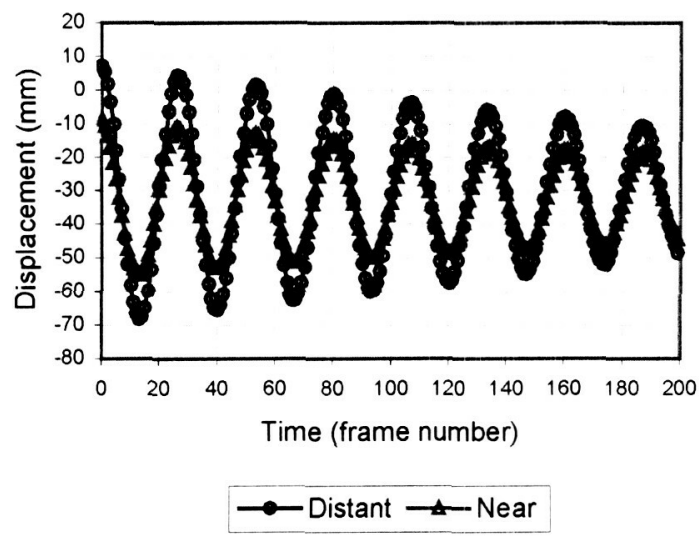

Figure 10 - Displacement of targets along the axis of pattern projection.

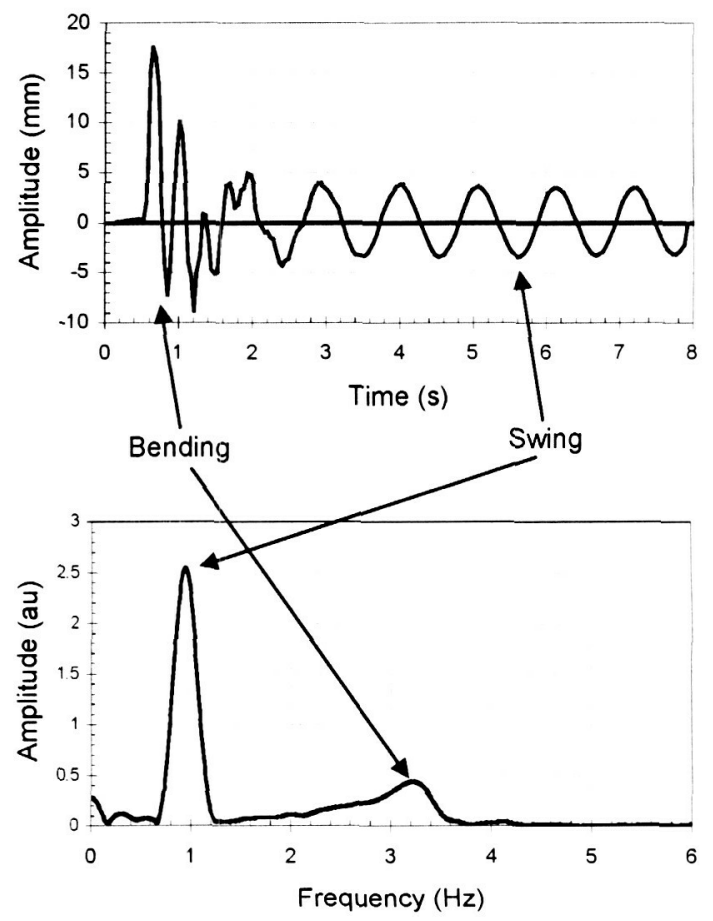

Figure 11 - Displacement of impulse excited transparent polymer in time and frequency domains. Notice the short-lived bending mode and the slowly decaying swinging mode.

experiment using this set-up. As with the first experiment, 200 image frames were acquired using the same equipment, and processed in the same way.

Figure 11 shows the displacement of one target in both the time and frequency domain, which clearly shows two dominant modes. The first is a one short lived bending oscillation at $3.18 \mathrm{~Hz}$ that settles into a more steady $0.94 \mathrm{~Hz}$ swinging mode. The reasons for the bending and swinging labels is apparent in Figure 12, which shows a plot of displacement amplitude at these specific frequencies. The bending mode has a maximum amplitude in the bottom-center of the structure and is induced directly from the impulse excitation at that location. However, this mode decays leaving the swinging mode that has an amplitude that varies in the vertical direction only and is proportional to the distance from the pivot axis.

Although this modal analysis is very basic, it suffices for the purposes of demonstrating the capabilities and potential of the measurement system. A more sophisticated modal analysis could be performed with the same acquired data, but it is not warranted in this case because we are not attempting to learn anything relating to the structure's properties. 


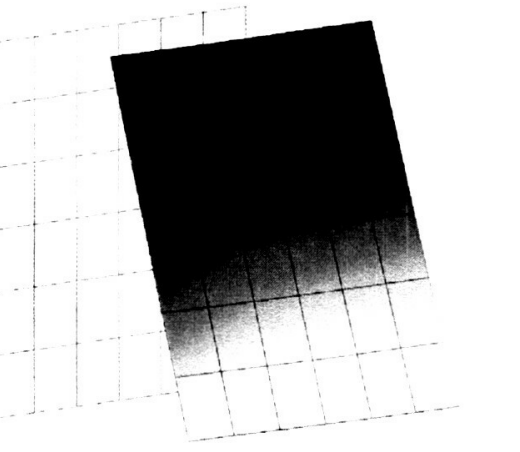

a) $0.94 \mathrm{~Hz}$ Swinging mode

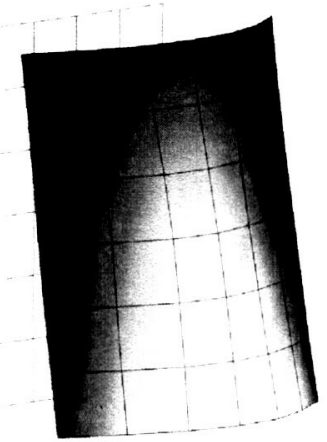

b) $3.18 \mathrm{~Hz}$ Bending mode

Figure 12 - Modal amplitudes across the measured area of the transparent membrane with reference to the stationary (planar) shape.

\section{Static Wrinkles in an aluminized polymer}

Because many gossamer space structures are aluminized for radiation protection, and in the case of solar sails, for thrust generation, the previously demonstrated measurements must be adapted for aluminized membranes. If the polymer is aluminized on only one side, LIFP measurements can be performed from the non-coated side. This actually offers the advantage of increased fluorescence intensity when compared to a transparent membrane (for a given illumination power and dye concentration). ${ }^{5}$ The increase in observable fluorescence intensity for aluminized membranes is due to reflections of both the laser and fluorescence light off of the membrane's aluminum back coating. Firstly, after passing though the polymer once, the laser is reflected by the aluminum coating and passes though the polymer a second time. This effectively doubles the illumination intensity causing a corresponding increase in generated fluorescence. Secondly, the fluorescence that would normally propagate in the direction of illumination and away from the observer is reflected by the aluminum coating back towards the observer, causing a further increase in detected fluorescence.

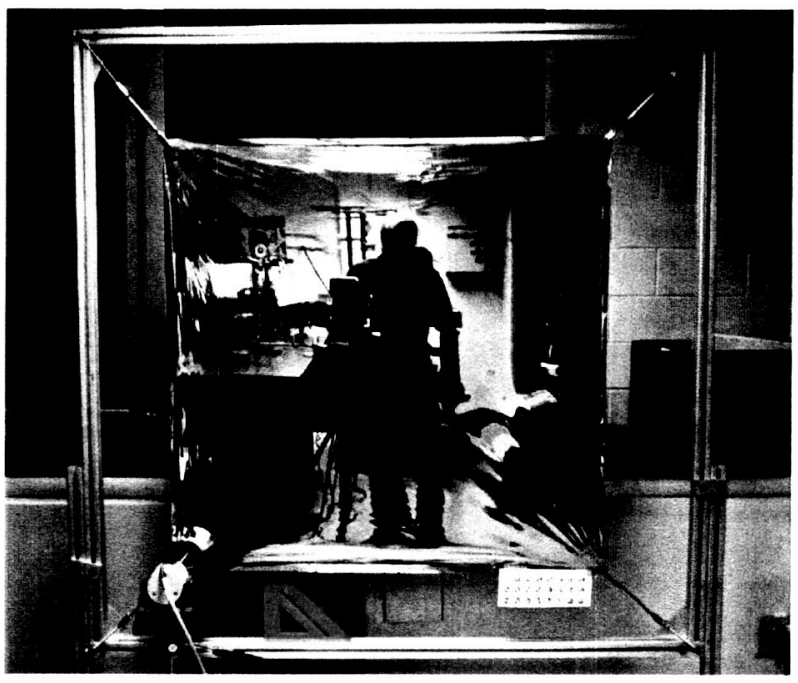

Figure 13 - Photograph of $1 \mathrm{~m}$ solar sail dye-doped polymer suspended in tension frame.

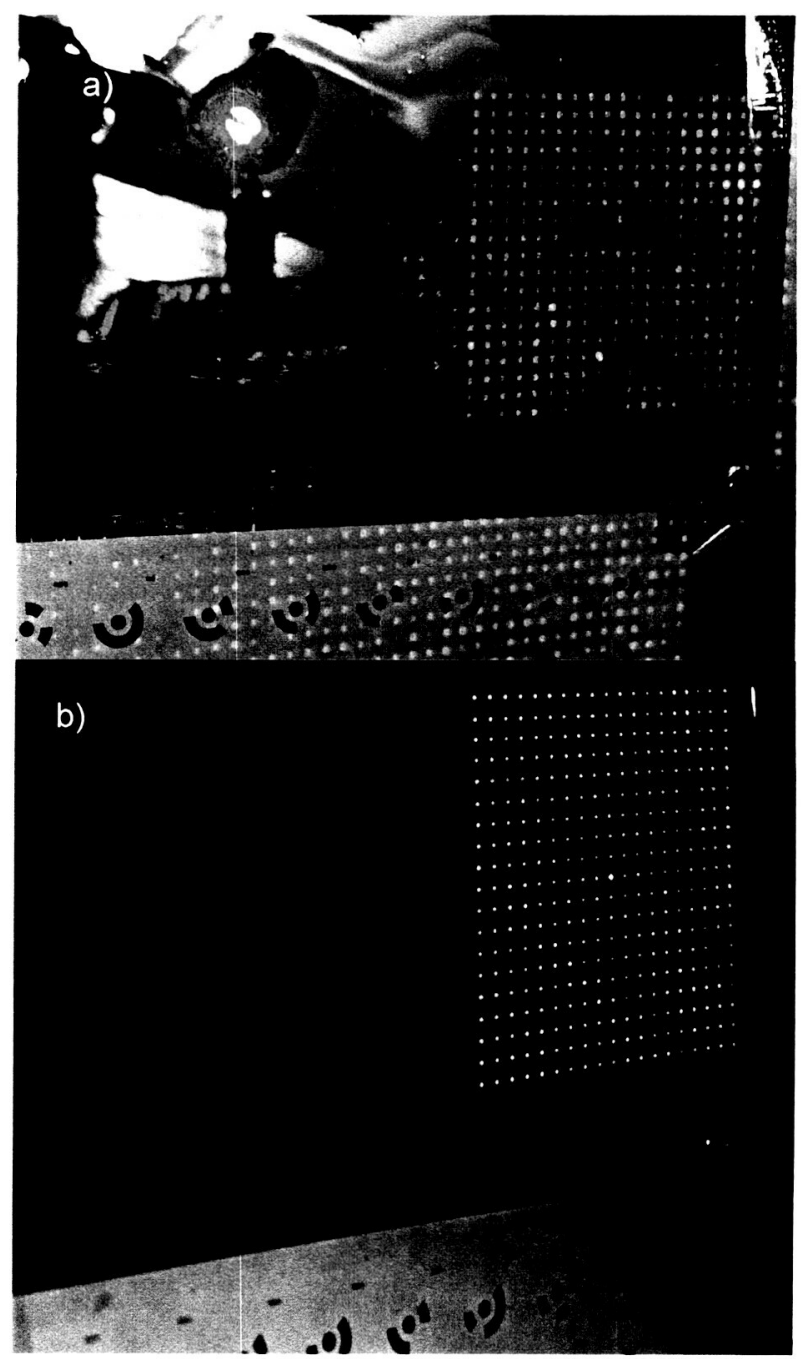

Figure 14 - Photographs of the 19-by-19 laser array projected onto the corner of the $1 \mathrm{~m}$ solar sail. Both an unfiltered image (a) and a filtered image showing only LIF (b) are shown 
To demonstrate the LIFP technique on aluminized polymers, we used a $1 \mathrm{~m}$ by $1 \mathrm{~m}$ model solar sail made from dye-doped CP1 polymer aluminized on one side only (SRS Technologies, Huntsville, AL). The polymer was reinforced on the edges and eyelets were placed in the corners to allow suspension in a tensioning frame, as illustrated in Figure 13. Tension was applied unevenly in the diagonal directions to encourage the formation of wrinkles in the corners. These wrinkles were then measured with a similar experimental set-up described earlier for the transparent polymer wrinkle measurements.

The area of measurement coverage can be seen in Figure 14a, which also show part of the projection setup reflected in the sail. The fluorescence is not evident in this image because it is swamped by the ambient light and incident laser light. It is also apparent from this image that the laser-projected spots are of insufficient quality (because of inconsistent shape and contrast) to be used as photogrammetric targets. An image of the same area viewed though an optical filter, Figure $14 \mathrm{~b}$, shows the significantly superior quality of the fluorescent targets. In these images, a set of coded targets is also visible below the membrane, which were used to help automate the photogrammetric processing and provide a scale reference.

Six images were acquired and processed to generate a 3D computer model representation of the wrinkled surface, shown in Figure 15. The computer model agrees with the actual surface by observation, and is of sufficient quality to clearly show wrinkles with amplitudes as low as $200 \mu \mathrm{m}$ peak-to-peak. These results demonstrate the potential of LIFP to perform high precision detailed examinations of (dye-doped) aluminized polymers.

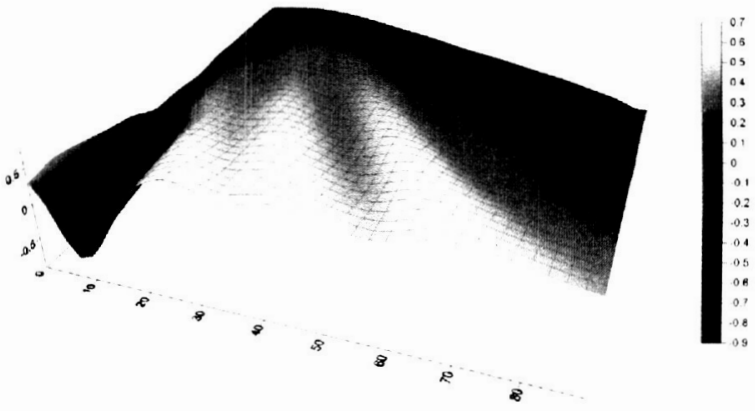

Figure 15 - Computer model of the surface wrinkling compiled from laser-induced fluorescence photogrammetric data. Scale in millimeters. The back corner was nearest to the eyelet.

\section{Dynamics of an aluminized polymer}

To demonstrate the capability of LIFP to be used for dynamic characterization of aluminized structures, the $1 \mathrm{~m}$ model solar sail membrane was also the subject of dynamic measurements. The experiment set-up used for the transparent membrane dynamic measurements described above was modified slightly to accommodate this larger structure. Again, a 7-by-7 spot array was projected onto the polymer and scaled to cover its entire surface. The polymer was then excited with a shaker driven by a random signal and video data was acquired at a rate of 20 frames per second.

The captured video data was photogrammetrically processed to provide out-of-plane displacement data, which was then processed further to determine vibrational modes. A spectral plot of the displacement averaged over every measurement point, shown in Figure 16, reveals several vibration modes. Four of the modes were chosen for further examination, and their mode shapes (shown in Figure 17) were reconstructed from the phase and amplitude information obtained

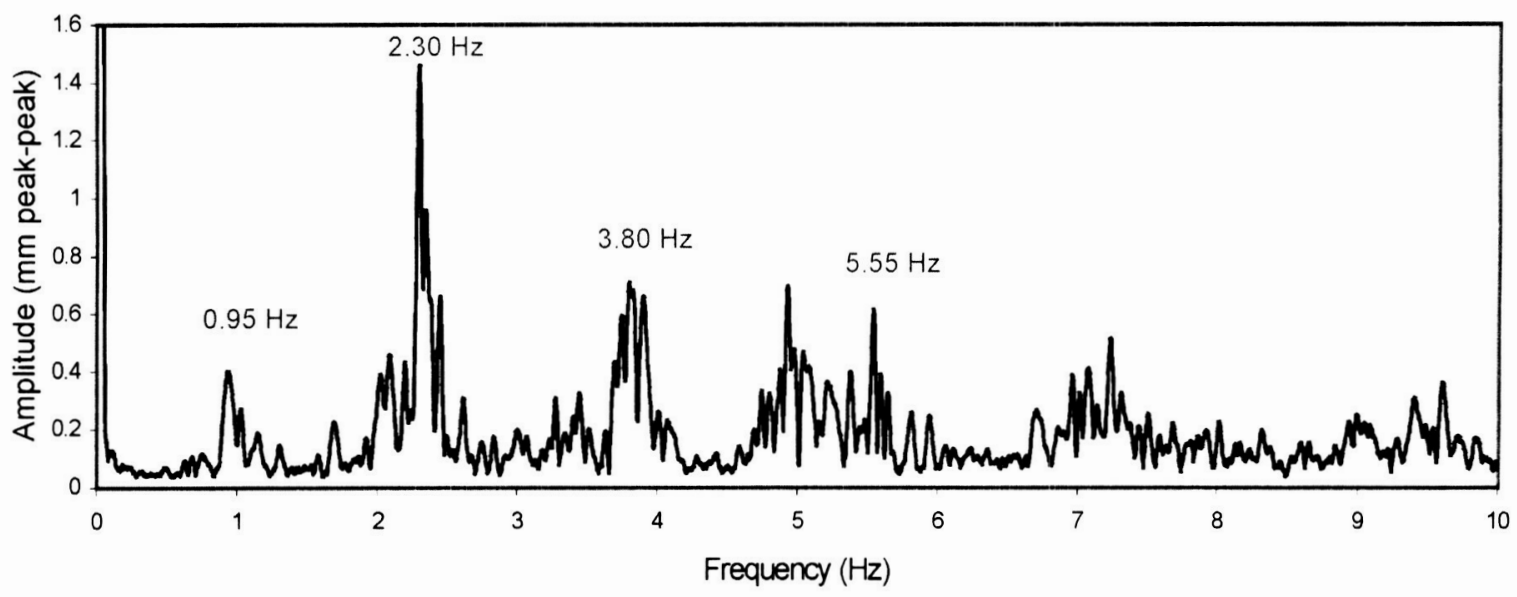

Figure 16 - Spectrum of out-of-place displacement for randomly excited $1 \mathrm{~m}$ solar sail averaged over every measurement point. 
from an FFT of each measurement point. A more rigorous modal analysis could be performed with the photogrammetry data, but in this case is not warranted. This is because the purpose is once again to demonstrate the measurement capability, and not to obtain specific information about the structural characteristics.

Although non-contact static measurements of aluminized membranes have been performed in the past, ${ }^{9}$ no dynamic measurements have yet been reported. This is because the long exposure times required for white-light dot projection methods prohibited dynamic measurements.

\section{Measurement Precision}

The precision obtained can be estimated by observing the apparent movement of stationary targets. In the dynamic measurement of the aluminized $1 \mathrm{~m}$ polymers, several stationary laser-induced fluorescent targets (both attached to and independent from the support frame) were used to establish a co-ordinate reference and scale. The image plane centroid data for these targets has been analyzed to determine the scatter in centriod determinations, which can be compared to other target generation techniques.

Table 1 details the $3 \sigma$ uncertainty (99\% confidence level) for each static target viewed by each of three cameras. The majority of these uncertainty values are well below one tenth of a pixel. Furthermore, the average uncertainty in the $\mathrm{X}$ dimension is 0.055 of a pixel and in the $\mathrm{Y}$ dimension 0.068 of a pixel, ignoring the outlier of Target 1 on Camera 3 . These values are comparable with other targeting techniques; ${ }^{11}$ hence the laser induced fluorescence target generation process itself does not appear to contribute any significant additional uncertainty.

Table $1-3 \sigma$ standard deviations of static target image plane coordinates (in pixels) measured in the aluminized $1 \mathrm{~m}$ polymer dynamic experiment.

\begin{tabular}{|c|c|c|c|c|c|}
\hline $\mathrm{X}$ & \multicolumn{5}{|c|}{ Target 1 Target 2 Target 3 Target 4 Target 5} \\
\hline & 0045 & ( & 0077 & 0.051 & 2 \\
\hline & 0. & 0. & 0.06 & 00 & 8 \\
\hline amera 3 & 0.044 & 0.043 & 0.095 & 0.064 & 0.043 \\
\hline
\end{tabular}

\begin{tabular}{|c|cccccc|}
\hline Y & Target 1 & Target 2 & Target 3 & Target 4 & Target 5 \\
\hline Camera 1 & 0.037 & 0.169 & 0.069 & 0.042 & 0.030 \\
Camera 2 & 0.041 & 0.038 & 0.057 & 0.077 & 0.040 \\
Camera 3 & 1.319 & 0.109 & 0.117 & 0.098 & 0.034 \\
\hline
\end{tabular}
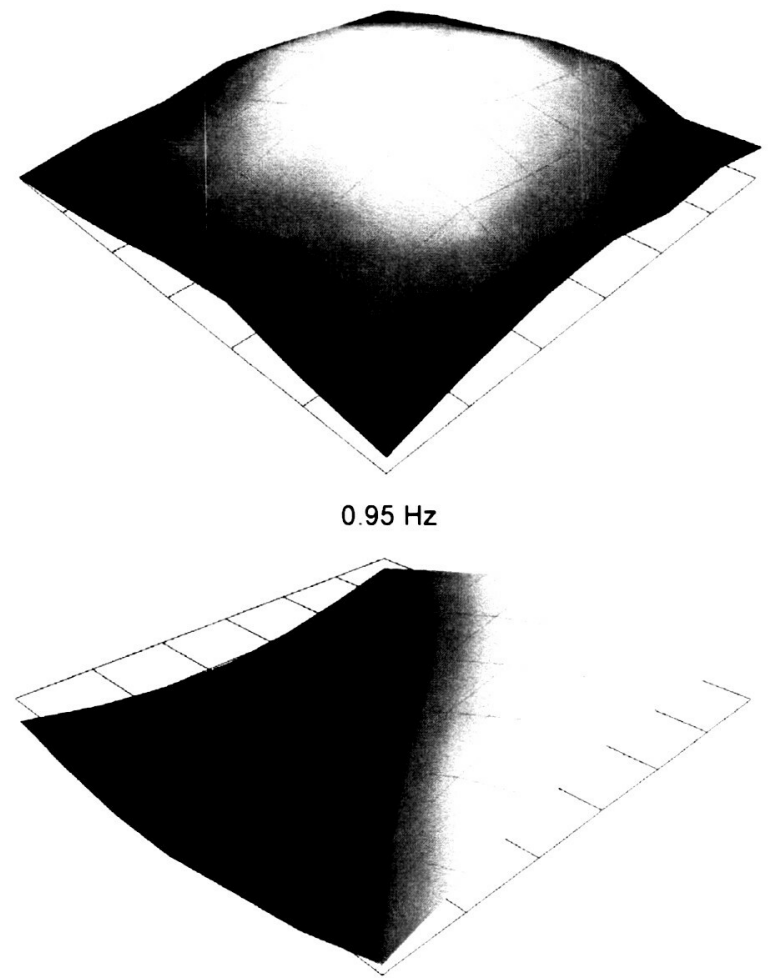

$2.30 \mathrm{~Hz}$

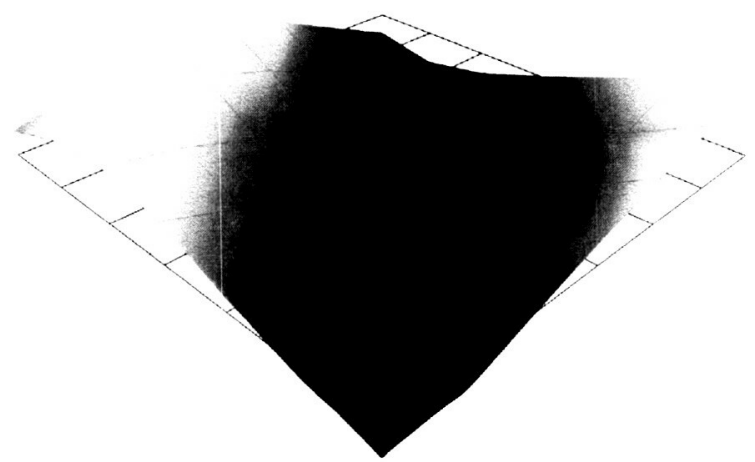

$3.80 \mathrm{~Hz}$

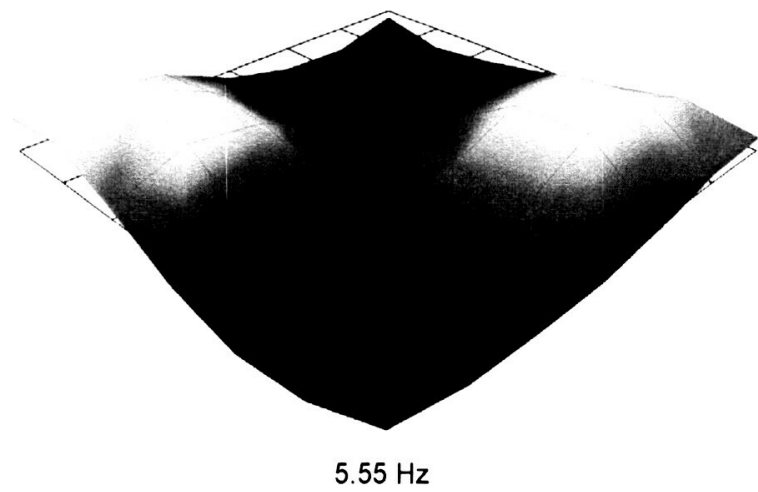

Figure 17 - A selection of mode shapes obtained from random excitation of the $1 \mathrm{~m}$ aluminized dye-doped polymer. The polymer was driven from the left corner. 


\section{Conclusion}

We have summarized a new laser-induced fluorescence photogrammetric target generation technique that has significant advantages over traditional targeting techniques when applied to gossamer space structures. Several demonstrations of the new target generation technique have been presented that illustrate its capability to perform both dynamic measurements and detailed high resolution surface profiling. Both the capability to measure transparent membranes and the ability to perform non-contact dynamic measurements of both transparent and aluminized membranes have not been previously reported. Furthermore, the precision of these measurements was found to be comparable to other photogrammetric measurements.

\section{$\underline{\text { References }}$}

1. Mikhail E. M., Bethel J. S., McGlone J. C., "Introduction to modern photogrammetry," John Wiley and Sons, New York, 2001.

2. Atkinson K. B. (editor), "Close range photogrammetry and machine vision," Whittles Publishing, Scotland, UK, 2001.

3. Pappa R. S., Black J. T., Blandino J.R., Jones T.W., Danehy P.M., Dorrington A.A., "Dotprojection photogrammetry and videogrammetry of gossamer space structures," to be published in the AIAA Journal of Spacecraft and Rockets, Vol. 40, No. 5, 2003.

4. Jones T.W., Dorrington A.A., Brittman P.L., Danhey P.M., "Laser induced fluorescence for photogrammetric measurement of transparent or reflective aerospace structures," Proceedings of the 49th International Instrumentation Symposium, Orlando Florida, May 2003.

5. Dorrington A.A., Jones T.W., Danehy P.M., "Laser-induced fluorescence photogrammetry for profiling gossamer space structures," Proceedings of the Conference on Lasers and Electro-optics 2003, Baltimore, Maryland, June 2003.

6. Burner A.W., Liu T., "Videogrammetric model deformation measurement technique," Journal of Aircraft, Vol. 38, No. 4, 2001, pp. 745-754.

7. Pappa R. S., Giersch L. R., Quagliaroli J. M., "Photogrammetry of a $5 \mathrm{~m}$ inflatable space antenna with consumer-grade digital cameras," Experimental Techniques, Society for
Experimental Mechanics, Vol. 25 No. 4, 2001, pp. 21-29.

8. Dharamsi U.K., Evanchik D.M., Blandino J.R., "Comparing photogrammetry with a conventional displacement measurement technique on a square Kapton membrane," AIAA paper 2002-1258, Proceedings of the 3rd AIAA Gossamer Spacecraft Forum, April 2002.

9. Pappa R.S., Jones T.W., Black J.T., Walford A., Robson S., and Shortis M.R., "Photogrammetry methodology development for gossamer spacecraft structures," AIAA Paper 2002-1375, Proceedings of the 3rd AIAA Gossamer Spacecraft Forum, April 2002.

10. Jones, T. W., Pappa, R. S., "Dot projection photogrammetric technique for shape measurements of aerospace test articles," AIAA Paper 2002-0532, Proceedings of the 40th AIAA Aerospace Sciences Conference, January 2002.

11. Black, J.T., Pappa, R.S., "Videogrammetry using projected circular targets: Proof-of-concept test," Proceedings of the 21 st International Modal Analysis Conference (IMAC), Kissimmee, Florida, February 2003. 